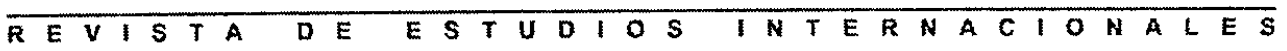

\title{
El área de libre comercio de las Américas: descripción, estado actual y perspectivas
}

\begin{abstract}
Esteban Tomic Errázuriz
Es un hecho bastante desconocido que en distintas cindades del continente, desde 1998, se reúnen cada tres meses 900 negociadores de 34 países para darle forma al ALCA (Área de Libre Comercio de las Américas), el más vasto mercado que haya conocido hasta hoy la humanidad, con 800 millones de personas y un producto interno de 12,5 millones de millones de dólares.

Muy pocos saben que desde julio de 2001 está disponible en Internet ${ }^{l}$ el resultado provisorio (borrador) de ese trabajo, que en 450 páginas sintetiza lo que 9 grupos han elaborado durante nuás de 500 dias de reuniones. Casi todos ignoran que desde 1994 se han ido cumpliendo, una tras otra, todas las etapas que fijaron para este proceso los Jefes de Estado y de Gobierno, rennidos en la Primera Cumbre Hemisférica de Miami, y que es altamente probable que el 1 de enero de 2005 todo ello comience a ser realidad, desde el Yukon hasta Tiera del Fuego.

El nuestro ha sido un proceso poco conocido, súbito y caudaloso, que contrasta, como lo hace también la geografia, con el desarrollo observado en Europa, que ha sido pausado, racionalmente planificado, altamente visible, dotado de instituciones y que ha obedecido a un diseño geopolitico original: impedir que en ese continente vuelva a darse una conflagración bélica mundial.
\end{abstract}

$\mathbf{E}$

s difícil explicar por qué nuestra América se mueve de esta manera.

Tal vez su historia es demasiado reciente: nuestras sociedades son hijas del Renacimiento y no experimentaron el len- to proceso de amalgama civilizatoria que fue la Edad Media. Por eso, seguimos imbuidos del ímpetu de los conquistadores, abriendo horizontes en el Nuevo Mundo, más a fuerza de voluntad y energía que de maña y paciencia.

${ }^{1}$ www.ftaa-alca.org. Asimismo, está disponible el primer compendio de legislación y regulación comercial hemisférica, al igual que todos los compromisos contraídos. 
Aunque posiblemente la explicación está también en las enormes diferencias en tamaño y poder entre los países que la integran. En Europa el eje franco-alemán, formado por dos países de dimensiones comparables, fue decisivo para llevar adelante la experiencia en las primeras décadas. En América no hay ningún país que pueda contrapesar el enorme poderío económico y militar de los Estados Unidos. Esta circunstancia se refleja en el proceso de toma de decisiones políticas, y la integración es, esencialmente, una decisión política.

Finalmente, otra causa podría ser que recién en los años noventa terminó de configurarse el cuadro político multilateral en el continente, cuando Canadá y los países caribeños que aún no lo habían hecho ingresaron a la Organización de los Estados Americanos (OEA), permitiéndoles jugar un rol principal en la escena internacional, y representar por vez primera al hemisferio en su conjunto.

Sean éstas u otras las causas del singular camino seguido por nuestros países, lo cierto es que llama la atención el hecho de que este enorme mercado no tenga padres identificables, como sí los tiene la Unión Europea en las personas de Robert Schuman y Jean Monnet, que la concibieron al término de la Segunda Guerra Mundial.

La integración en América no es sólo inaparente y tumultuosa, sino además muy reciente.

En efecto, si consideramos los 12 acuerdos de Unión Aduanera o de Libre Comercio actualmente vigentes entre países de la región, sólo dos se remontan a los años sesenta, uno a los setenta, y todos los demás se firmaron y entraron en vigor en la década de los noventa o a inicios de este siglo.

Tardó nuestro continente en percibirse a sí mismo como entidad con perfil e intereses propios. En el plano retórico tal vez lo hizo, pero cuando se trató de pasar a los hechos, ya que en eso consiste crear un Mercado Común, una Unión Aduanera o una Zona de Libre Comercio, la cosa se tornó muy difícil, casi impracticable.

\section{Nuestro continente tardó en percibirse como entidad con perfil e identidad propios.}

Conocido es, por ejemplo, el rechazo que encontró en los círculos de gobierno y privados de los Estados Unidos la decisión de cinco países sudamericanos de constituir, en 1969, un espacio económico común, el Pacto Andino. Otro tanto puede decirse de la iniciativa de CECLA, que en esos mismos años dio lugar a un público desacuerdo entre Richard Nixon y los cancilleres latinoamericanos que llegaron a presentarle la iniciativa.

Recién con el término de la Guerra Fría se abrió el coloso del norte a la posibilidad de conformar acuerdos de integración. El Acuerdo de Libre Comercio de América del Norte (NAFTA), del cual forman parte Estados Unidos, México y Canadá, nació el mismo año en que se colocó la Asociación de Libre Comercio de las Américas (ALCA) en la agenda continental. 


\section{El Proceso de Cumbres, el ALCA Y LA OEA}

En la primera Cumbre de las Américas, que tuvo lugar en Miami en diciembre de 1994, los gobernantes de treinta y cuatro de las treinta y cinco naciones del hemisferio occidental (todas ellas salvo Cuba) acordaron lo siguiente:

"Nuestro progreso económico continuo depende de políticas económicas sólidas, del desarrollo sostenible y de un sector privado dinámico. Una clave para la prosperidad es el comercio sin barreras, sin subsidios, sin prácticas desleales $y$ con un creciente flujo de inversiones productivas. La eliminación de los obstáculos para el acceso al mercado de los bienes y servicios entre nuestros países promoverá nuestro crecimiento económico. Una economía mundial en crecimiento aumentará también nuestra prosperidad interna. El libre comercio y una mayor integración económica son factores clave para elevar el nivel de vida, mejorar las condiciones de trabajo de los pueblos de las Américas y proteger mejor el medio ambiente. Por consiguiente, decidimos iniciar de inmediato el establecimiento del 'Área de Libre Comercio de las Américas' en la que se eliminarán progresivamente las barreras al comercio y la inversión. Asimismo, resolvemos concluir las negociaciones del 'Área de Libre Comercio de las Américas' a más tardar en el año 2005, y convenimos en alcanzar avances concretos hacia el logro de este objetivo para el final de este siglo".

En esa ocasión, los jefes de Estado y de Gobierno democráticamente elegidos del hemisferio occidental firmaron una declaración de principios titulada "Pacto para el Desarrollo y la Prosperidad de las Américas" y un Plan de Acción.

Los objetivos de la alianza fueron definidos como:

- La preservación y el fortalecimiento de la comunidad de las democracias en las Américas.

- La promoción de la prosperidad a través de la integración económica y el libre comercio.

- La erradicación de la pobreza y la discriminación en el hemisferio.

- La garantía de un desarrollo sostenible así como de la conservación del medio ambiente para las generaciones futuras.

Para el logro de estos cuatro objetivos básicos los jefes de Estado y de Gobierno aprobaron un Plan de Acción que contiene 23 iniciativas, una de las cuales es la creación del ALCA.

El proceso de Cumbres Hemisféricas, de las que se han realizado tres (Miami 1994, Santiago 1998 y Quebec 2001), es el origen y el motor de un vasto proceso de acercamiento entre los países de la región, impulsados no sólo por una lógica económica, sino también por necesidades estratégicas vinculadas a la seguridad colectiva.

\section{Las Cumbres Hemisféricas obedecen a la lógica económica y a necesidades estratégicas vinculadas con la seguridad colectiva.}

De las 23 iniciativas antes mencionadas, el ALCA es la más importante. 
Es interesante observar que la concepción inicial de este proceso no contemplaba un rol para la Organización de los Estados Americanos, el principal órgano político del hemisferio. La razón radicaba en la poca confianza que había en la Organización.

La OEA, fundada en 1948 en plena Guerra Fría, tuvo durante mucho tiempo fama de ser un instrumento al servicio de los Estados Unidos. Sólo a comienzos de los años noventa comenzó a recuperar prestigio y lustre, pero éste era un desarrollo demasiado reciente como para que las Cumbres se apoyaran en ella de manera más decidida en sus inicios.

Sin embargo, los ocho años transcurridos muestran una creciente convergencia entre el proceso de Cumbres y la OEA, al punto de que se ha resuelto que, a partir de 2002, la Asamblea General anual de la Organización sea también la ocasión en que los ministros de Relaciones Exteriores examinen el avance del proceso de Cumbres.

\section{Hay creciente convergencia entre el proceso de Cumbres Hemisféricas y la OEA.}

La OEA se ha convertido en la práctica en la Secretaría Técnica de las Cumbres y desempeña, como veremos, un activo rol de soporte de las negociaciones del ALCA.

\section{Desarrollo del ALCA}

Desde su inicio en 1994 se pueden observar varias fases en el desarrollo del ALCA.

\section{a) Fase preparatoria:}

Va desde diciembre de 1994 hasta abril de 1998, entre la Primera y la Segunda Cumbre Hemisférica de jefes de Estado y de Gobierno.

En esta fase, el ALCA ya tenía sus mecanismos de ejecución claramente definidos: las reuniones ministeriales, las reuniones vice ministeriales, los grupos de trabajo hemisféricos, el Comité Tripartito (integrado por la Organización de los Estados Americanos, el Banco Interamericano de Desarrollo y la Comisión Económica de las Naciones Unidas para América Latina y el Caribe).

Tal como se instruye en el Plan de Acción, los ministros responsables de comercio definieron el programa de trabajo técnico a los efectos de preparar las negociaciones.

En la cuarta reunión ministerial, en San José de Costa Rica, en marzo de 1998, los 34 ministros convinieron en la Declaración de San José, en la que se definieron los objetivos y principios generales del ALCA, los objetivos específicos por áreas temáticas, así como la estructura, la organización y otros aspectos sustantivos y administrativos que iban a orientar las negociaciones. Los ministros acordaron también recomendar a los jefes de Estado y de Gobierno la iniciación de las negociaciones formales del ALCA en la Segunda Cumbre de las Américas, a celebrarse en Santiago de Chile en abril de 1998.

Esta recomendación se reflejó en la Declaración de la Segunda Cumbre de las Américas, en la que se pusieron en marcha formalmente las negociaciones. 
b) Fase de negociaciones:

Ésta se subdivide, a su vez, en dos primeras subfases ya cumplidas, de 18 meses de duración cada una: la primera se extendió desde la Cumbre de Santiago a la Quinta Reunión Ministerial de Comercio celebrada en Toronto, Canadá, en noviembre de 1999, y la segunda desde esta fecha hasta la Sexta Reunión Ministerial de Comercio, celebrada el 7 de abril de 2001 en Buenos Aires, Argentina.

El resultado concreto de esta tarea ya cumplida es el borrador del acuerdo que mencionábamos al inicio, y la adopción por los países de 18 medidas específicas de facilitación de negocios que, sin ser parte del ALCA, tienen una gran incidencia en el menor costo y la fluidez del comercio transfronterizo.

Una tercera subfase de las negociaciones, actualmente en curso, va de abril 2001 a octubre 2002 , mes en que se celebrará la Séptima Reunión Ministerial de Comercio en Ecuador. El resultado de este trabajo debería ser un segundo borrador del acuerdo del ALCA. La subfase final se extenderá de esta fecha hasta diciembre de 2004 bajo la co-presidencia de Brasil y los Estados Unidos.

\section{ESTRUCTURA. DEL MECANISMO DE NEGOCIACIÓN}

La estructura acordada para las negociaciones consiste en la presidencia rotativa del proceso negociador, un calendario fijo de fechas y lugares para las reuniones ministeriales en todo el período que concluye el 31 de diciembre de 2004; un Comité de Negociaciones Comerciales a nivel de viceministros y nueve grupos de negociación.

También se ha acordado que las reuniones de los grupos de negociación tengan lugar en un único lugar que rota del modo siguiente: Miami (desde el 1 de mayo de 1998 al 28 de febrero de 2001), Ciudad de Panamá (1 de marzo de 2001 a 28 de febrero de 2003), y Ciudad de México (1 de marzo de 2003 a 31 de diciembre de 2004).

Se creó una secretaría administrativa encargada de proporcionar apoyo logístico y administrativo para las negociaciones, prestar servicios de traducción de documentos e interpretación, llevar la documentación oficial de las negociaciones y publicar y distribuir documentos. La secretaría administrativa está localizada en el mismo sitio en que se realizan las reuniones de los grupos de negociación.

\section{El Comité de Negociaciones \\ Comerciales del ALCA cumple el papel central en su desarrollo.}

El Comité de Negociaciones Comerciales cumple el papel central en la gestión de las negociaciones del ALCA. Esto fue reafirmado por los ministros de Comercio en su Declaración de Toronto (noviembre de 1999), en la que se señala que el Comité debe orientar la labor de los grupos de negociación y de los otros tres grupos y comités creados por la Declaración de San José; así como asegurar la transparencia en las negociaciones; supervisar a la secretaría administrativa; supervisar la puesta en práctica de medidas de 
facilitación de negocios acordadas e identificar fuentes de financiamiento para su implementación; abordar asuntos que los grupos de negociación no hayan podido resolver y asegurar que se realizan avances en todas las áreas y grupo de negociación. El Comité debe reunirse por lo menos tres veces entre reuniones ministeriales consecutivas, o con más frecuencia si es necesario.

Los nueve grupos de negociación son: acceso a mercados (que abarca medidas arancelarias y no arancelarias, procedimientos aduaneros, salvaguardias, reglas de origen y normas y barreras técnicas al comercio); inversión; servicios; compras del sector público; solución de controversias; agricultura, propiedad intelectual; subsidios; antidumping y derechos compensatorios, y política de competencia. Se crearon asimismo tres Comités No Negociadores Especiales: un Grupo Consultivo sobre Economías Más Pequeñas; un Comité de Representantes Gubernamentales sobre la Participación de la Sociedad Civil y un Comité Conjunto de Expertos del Sector Público y Privado en Comercio Electrónico.

Los tres Grupos y Comités Especiales No Negociadores reflejan las preocupaciones e intereses de muchos participantes en sus respectivas áreas del proceso ALCA. Tal como se especifica en la Declaración de San José, la función del Grupo Consultivo sobre Economías Más Pequeñas consiste en "seguir el proceso del ALCA, evaluando las inquietudes e intereses de las economías más pequeñas" y "elevar a la consideración del Comité de Negociaciones Comerciales los temas de interés para las economías más pequeñas y hacer las recomendaciones para abordar estos temas".

\section{Los Comités Especiales reflejan} las preocupación de muchos participantes en el proceso del ALCA.

Reconociendo los intereses y preocupaciones de diversos sectores de la sociedad en relación con el ALCA, los ministros establecieron el Comité de Representantes Gubernamentales sobre la Participación de la Sociedad Civil y le solicitaron que recibiera los aportes de ella, que los analizara y que presentara la gama de opiniones para la consideración de los ministros. La misión del Comité Conjunto de Expertos del Sector Público y Privado en Comercio Electrónico consiste en examinar varios temas que guardan relación con ese comercio, incluido el examen del grado de preparación para Internet de los países que participan en el ALCA y la manera de tratar el comercio electrónico en el contexto de las negociaciones.

Al Comité Tripartito, formado por la OEA, el BID y la CEPAL, se le solicitó que contribuyera con apoyo técnico, analítico y logístico al proceso del ALCA, así como con asistencia técnica a los países participantes. En la práctica, este apoyo ha incluido ayudar a los gobiernos a compilar inventarios de leyes y reglamentos nacionales y regionales así como bases de datos sobre comercio y aranceles aduaneros, analizar normas y disposicio- 
nes regulatorias vigentes relacionadas con el comercio, y preparar estudios y documentos de antecedentes relativos a los ámbitos de negociación. Otras funciones del Comité Tripartito han consistido en dar respaldo a las presidencias de los diferentes grupos conforme a sus solicitudes; el trabajo con la secretaría administrativa en la labor de coordinar la traducción, las fechas de presentación y la distribución de documentos para las reuniones; la administración del sitio oficial del ALCA en la Internet en nombre de los países; el financiamiento de la secretaría administrativa, así como la realización de actividades de capacitación y educativas en beneficio de los países.

El trabajo del Comité Tripartito, que ha acompañado el proceso desde su inicio, es considerado un modelo de cooperación interagencias.

Así como la existencia de un amplio contingente de negociadores ha servido para crear una muy valiosa red de conocimientos técnicos y relaciones interpersonales que en definitiva contribuyen a acercar a los países entre sí, otro tanto ha ocurrido con la colaboración que han desarrollado en materia ALCA los tres organismos que conforman el Comité Tripartito.

\section{LA MARCHA DE LAS NEGOCIACIONES}

Los negociadores lograron avances importantes bajo la presidencia de Canadá en la primera fase de las negociaciones, es decir, en el período de 18 meses comprendido entre mayo de 1998 y no- viembre de 1999, fecha de la Quinta Reunión Ministerial, celebrada en Toronto, Canadá. En esta reunión, los ministros evaluaron el progreso de las negociaciones durante la primera fase como "considerable".

A partir de septiembre de 1998, cada uno de los nueve grupos de negociación se ha venido reuniendo regularmente. Como se dijo, cada ronda de reuniones de grupos de negociación congrega a más de 900 negociadores comerciales de los 34 países participantes y constituye un extraordinario esfuerzo de determinación política, capacidad técnica y posicionamiento estratégico de las naciones participantes. Del proceso han surgido bases de datos completas, compendios de leyes y regulaciones y otra información de antecedentes necesaria para las negociaciones. Este proceso ha mejorado enormemente la transparencia en cuanto a las reglas comerciales y de mercado vigentes en el hemisferio.

\section{En Canadá el avance en las negociaciones fue evaluado como "considerable".}

Finalmente, es importante señalar que varios subgrupos de países hablan con una sola voz en la mesa de negociaciones. Esto incluye a la Comunidad Andina, al Mercosur y a CARICOM. Cada uno de esos grupos subregionales presenta sus posiciones conjuntamente, lo que es precedido por intensas consultas entre sus miembros. Recientemente, la región del Caribe creó una nueva estructura para 
formular y dar a conocer las opiniones de los países de la CARICOM en materia de negociaciones comerciales internacionales, y este Mecanismo Negociador Regional del Caribe habla en el ALCA en nombre de sus miembros.

\section{La Comunidad Andina, el Mercosur} y la CARICOM participan con una sola voz en las negociaciones del ALCA.

\section{ESTADO ACTUAL DE LAS NEGOCIACIONES}

A medida que se aproxima el término del plazo, las negociaciones se vuelven más difíciles, porque se han concentrado en torno a los temas verdaderamente complicados.

Uno de ellos es cómo establecer las equivalencias entre las tarifas aduaneras. Otro es el de las compras de gobiemo. También hay que resolver si los servicios serán objeto de listas positivas (enumeración de los que se pueden prestar) o negativas (de los que no se pueden prestar). Todas éstas son decisiones indispensables para definir las reglas de acceso al mercado.

El 15 de mayo se retomaron en Panamá las negociaciones que fueron suspendidas sin resultados, semanas antes, en Isla Margarita (Venezuela). El propósito es presentarles a los ministros un nuevo texto en su reunión de octubre próximo en Ecuador.

A las dificultades enunciadas se suma la aún incierta aprobación definitiva del TPA (Trade Promotion Authority) en el congreso norteamericano. Ello, porque el TPA también define parámetros para los negociadores de Estados Unidos.

Con todo, existe confianza en que de aquí a octubre habrá humo blanco y en que las negociaciones de acceso al mercado se iniciarán como está previsto.

\section{UN BALANCE PROVISIONAL DEL ALCA}

Señalábamos al comienzo que el proceso de integración de las economías americanas es muy reciente. Data de términos de la Guerra Fría o, puesto en otros términos, de comienzos de la globalización.

Sin desconocer la importancia que han tenido un puñado de estadistas que, puestos a pensar en las futuras generaciones, optaron por enfrentar la inercia y crear un marco de relaciones dotado de nuevas instituciones y valores, puede afirmarse que el proceso ha sido más bien de carácter aluvional. El sucederse de los acontecimientos a nivel mundial y regional hizo que los factores se combinaran dando lugar a la irrupción de este nuevo elemento que es la integración.

Desde el punto de vista de la teoría del centro y la periferia, ello equivale a que una parte de la periferia ha sido invitada a integrarse al centro, previo establecimiento de reglas del juego y la adopción de políticas que implican profundas reformas estructurales.

En tal sentido, es sintomático el caso de Chile: ubicado, con relación a los Estados Unidos y Europa en la más apartada periferia, acaba de acordar con ésta 
última una asociación que le garantiza una relación política y de cooperación privilegiada y, a partir del 1 de enero de 2003, el acceso libre al mercado europeo (400 millones de personas en la actualidad) de una parte sustancial de sus exportaciones. Es probable que concluya también este año otro acuerdo semejante con los Estados Unidos.

Gracias a que oportunamente abrió su economía al mundo, y también a que su orden social presenta índices comparables con los del centro en materia de democracia, respeto a los derechos humanos, transparencia, probidad, regulación de la competencia, mercado de capitales y otros, nuestro país se adentra en el siglo que se inicia en calidad de socio de un centro de acceso limitado, que seguirá siendo el vórtice de donde proviene el dinamismo que mueve la economía del mundo.

Lo que vale para Chile hoy debería regir a partir de 2005 para todos los países de América que negocian los compromisos del ALCA.

Esto implica una revolución sin precedentes, tan nueva y tan súbita, que apenas comienza a ser analizada en sus aspectos positivos y negativos.

Para examinar estos aspectos es necesario hacer diferencias entre las distintas subregiones.

Los compromisos del ALCA implican una revolución cuyos aspectos recién comienzan a analizarse.
Centroamérica tiene un gran interés en obtener un acceso garantizado al mercado de los Estados Unidos, que es actualmente el destino principal de sus exportaciones. Esto vale en particular para sus productos agrícolas, textiles y para los procesos de maquila industrial. Es muy importante asegurarles a estos últimos el ingreso al mercado norteamericano antes de que China se abra paso con fuerza en el mercado internacional.

Desde el punto de vista de las inversiones, Centroamérica quiere recibir industrias de tecnología avanzada, como es el caso de Intel en Costa Rica, por lo cual tiene interés en el capítulo de Propiedad Industrial.

El Caribe concentra su atención en los servicios, entre los cuales el turismo y las comunicaciones son relevantes. Estas últimas, porque pueden dar lugar a una industria exportadora de procesamiento de datos, aprovechando la circunstancia de que se trata de países mayoritariamente anglófonos.

Los países andinos también son altamente dependientes de las exportaciones a los Estados Unidos, aunque éstas presentan una diversificación mayor. A la vez, tienen mucho interés en recibir inversiones de aquel país que les permitirían modernizar su infraestructura (transporte, telecomunicaciones, sanitarias).

El Mercosur es, tal vez, el menos entusiasta de los negociadores del ALCA. Comercia en alto grado con la Unión Europea, posee una gama muy variada de productos de exportación y compite con los Estados Unidos en una serie de rubros, especialmente agrícolas (azúcar, alcohol, jugo de naranja, leche, carne, fruta). 
Tal vez por ello Brasil se aseguró la co-presidencia, junto con los Estados Unidos, en la fase final de la negociación.

Chile ya cruzó el umbral de estas dudas cuando en la Cumbre de Miami aceptó incorporarse al NAFTA como "el cuarto amigo". Ello aún no se concreta, pero en los ocho años transcurridos ha profundizado su política de apertura a los mercados con éxitos tan notables como los acuerdos con México, Canadá, APEC y recientemente la Unión Europea.

México y Canadá, sobre todo el primero, son, en teoría, los países menos interesados en que el ALCA sea una pronta realidad. Ello, porque ya gozan de acceso libre al mercado norteamericano debido a su pertenencia al NAFTA.

Los Estados Unidos, por su parte, proceden en la materia con el alma dividida; por un lado fueron ellos los que tomaron la iniciativa en la Primera Cumbre Hemisférica, y todos sus gobiemos desde entonces han mantenido en pie el ofrecimiento del libre comercio en las Américas. Por el otro lado, el gobierno, al otorgar subsidios y protección a sectores clave como la agricultura y el acero, y el Congreso, al negarle al presidente la facultad de negociar los acuerdos sin ulterior modificación por la rama legislativa, adoptan medidas totalmente contrarias a dicho propósito.

Independiente de los puntos de vista nacionales o regionales, un análisis objetivo indica que el ALCA indudablemente incidiría en una mejoría de las condiciones económicas en el hemisferio.

Una inversión más racional de los recursos promovería sistemas de produc- ción más eficientes. La tecnología y el capital tendrían gran movilidad a través de las fronteras y las diferencias en el ingreso per cápita entre las naciones tenderían a disminuir, al igual que los precios finales de muchos bienes y servicios. Los consumidores se verían favorecidos en la medida en que sus salarios se elevarían, mientras el nivel de los precios se mantendría estable.

\section{Pese a que tomaron la iniciativa, los Estados Unidos han adoptado medidas contrarias al libre comercio en las Américas.}

Ello no quiere decir, por supuesto, que todos se beneficiarían, o que todos los países tendrían igual provecho del ALCA.

Algunos sectores en determinados países verían deteriorarse su posición, ya que las relaciones comerciales se desplazarían dramáticamente. Industrias ineficientes, desprovistas de protección, tenderían a desaparecer, mientras aquellas eficientes tenderían a crecer rápidamente, gracias al nuevo acceso a los mercados externos.

En los Estados Unidos, por ejemplo, sectores como el azúcar, los jugos de fruta, los textiles, y el acero disminuirían su tamaño. Simultáneamente, los sectores de computadores y de telecomunicaciones se expandirían.

En el resultado final, tanto los Estados Unidos como sus socios del ALCA llegarían a la conclusión de que se ha producido una ganancia neta de bienestar. 


\section{BIBLIOGRAFÍA}

Audley, Jorge y Sherwin, Edward. Politics and Parallel Negotiations. Environment and Trade in the Western Hemisphere, Carnegie Endorvment, Global Policy Program, November 25, 2001-April 2002.

Casanueva Ojeda, Héctor. "Europa, América Latina y la Embajador de Chile Competencia Mundial, ante ALADI", El Mostrador, 19 de abril de 2002.

"La Integración Europea es un Referente", El Mostrador, 9 de mayo de

Devlin, Robert y Estevadeordal, Antoni. The FTAA: Some Longer Term Issuesy Garay, Jorge Luis INTAL-ITD, August 1999

Gaviria, César. "The Doha Development Agenda Secretario General de la OEA and the FTAA Process. The Challenge of Trade Capacity Building", Introductory Remarks, February 28, 2002.

Saéz Rojas, Alejandro. "Economía. Su antidogma para enfrentar la Globalización. La luz roja de la CEPAL", El Mercurio, 4 de mayo de 2002.

Salazar-Xirinacs, José M. "El ALCA a la Mitad del Camino. Avances Director, Unidad de Comercio, OEA hasta hoy", Retos y Perspectivas, 12 de noviembre de 2001.

"El proceso del ALCA: de Miami 1994 a Quebec 2001", en José M. Salazar- Xirinacs y Maryse Roberts (eds.), Hacia el Libre Comercio en las Américas, (2001).

"Key Issues in Resource Mobilization for Trade Capacity Building", OAS, Hall of the Americas, February 28, 2002.

"After Quebec and Half Way Through to 2005", Inter-American Dialogue, May 10, 2002.

Thery, Jane. "Business Facilitation: Concrete Progress Subdirectora", Oficina de Seguimiento in the FTAA Process de Cumbres, OEA Trade Unit, March 2001.

El autor agradece los comentarios del profesor James Ferrer, director del CLAI (Center for Latin American Issues, GWU) y de Jane Thery, subdirectora de la Oficina de Seguimiento de Cumbres de la OEA. 